\title{
Nuclear exclusive vector meson photoproduction
}

\author{
V.P. Gonçalves ${ }^{1}$, M.V.T. Machado ${ }^{2,3}$ \\ 1 Instituto de Física e Matemática, Universidade Federal de Pelotas, Caixa Postal 354, CEP 96010-090, Pelotas, RS, Brazil \\ 2 Universidade Estadual do Rio Grande do Sul - UERGS, Unidade de Bento Gonçalves, CEP 95700-000, Bento Gonçalves, \\ RS, Brazil \\ 3 High Energy Physics Phenomenology Group, GFPAE IF-UFRGS, Caixa Postal 15051, CEP 91501-970, Porto Alegre, RS, Brazil
}

Received: 17 April 2004 / Revised version: 21 September 2004 /

Published online: 18 November 2004 - (C) Springer-Verlag / Società Italiana di Fisica 2004

\begin{abstract}
We calculate the nuclear cross section for vector meson exclusive photoproduction within the QCD color dipole picture and in the Regge approach. For the former approach, we have considered the phenomenological saturation model, whereas for the latter we use a model based on the dipole pomeron framework. Theoretical estimates for scattering on both light and heavy nuclei are given over a large range of energy.
\end{abstract}

\section{Introduction}

Exclusive vector meson production by real and virtual photons is an outstanding process providing important information on the transition region from the soft dynamics (at low virtualities of the photon $Q^{2}$ ) to the hard perturbative regime at high $Q^{2}[1,2]$. In principle, a perturbative approach is only justified if a hard scale is present in the process, e.g. the photon virtuality and/or a large mass of the vector meson. For photoproduction of light mesons, such a scale is not present and one has to rely on non-perturbative models. In general, a simple Regge pole phenomenology, with a soft pomeron having intercept larger than one is enough to describe the energy dependence of the meson cross section at the present accelerators. In some pQCD approaches, as the saturation model, even this soft process can be described, where the transition is set by the saturation scale. Both models give an effective pomeron intercept increasing with photon virtuality and meson mass. For $\phi$ and $J / \Psi$ mesons, the pomeron intercept is considerably large and consistent with that one obtained in the usual pQCD approaches. Despite the good agreement for the currently available energies, an extrapolation to higher energies of the experimental fits implies a large growth for the cross section which would violate the unitarity at sufficiently high energies. Therefore, dynamical modifications associated to the unitarity corrections are also expected to be present in this process $[3,4]$. Moreover, these effects should be enhanced in nuclear processes $[5,6]$. In particular, in the planned $e A$ colliders at HERA and RHIC, the experimental analyses of the exclusive vector meson production could be very useful to constrain the QCD dynamics $[7,8]$.

Our goal in this paper is to investigate the high energy vector meson exclusive photoproduction on nuclei. In particular, we improve the previous analyses of vector meson production [9] which are based on an extrapolation of the DESY-HERA experimental fits for the proton case. Here, we will consider unitarized cross sections ab initio. In order to do so, we consider two distinct and well established theoretical scenarios which do not violate the unitarity bound in the asymptotic regime to be probed in future colliders. This allows us to analyze the nuclear vector meson protoproduction as a potential process to discriminate between these different theoretical approaches. First, we consider the color dipole description of the $\gamma A \rightarrow V A$ ( $V=\rho, \omega, \phi, J / \Psi)$ process, which is quite successful for the proton case $[3,4]$ and can be extended to nuclear targets via the Glauber-Gribov formalism. It is important to refer to the pioneering papers [10-14], where these issues were first addressed and their further developments [15-17] as well. Related calculations in the $k_{\perp}$-factorization approach (the dipole approach is equivalent to it at leading logarithmic approximation) can be found in [18-21]. In the color dipole approach, the degrees of freedom are the photon (color dipole) and meson wavefunctions as well as the dipole-nuclei cross section. Such an approach enables one to include nuclear effects and the parton saturation phenomenon. The latter one is characterized by a typical momentum scale $Q_{\text {sat }}$ (saturation scale) and it has been constrained by experimental results in deep inelastic scattering (DIS) and diffractive DIS [22]. Here, we will use an extension of the phenomenological saturation model for nuclear targets [23]. This model reasonably describes the experimental data for the nuclear structure function and has been used to predict the nuclear inclusive and diffractive cross sections for heavy quark photoproduction [24]. The nuclear saturation scale, $Q_{\mathrm{s} A}$, provides the transition between the color transparency and the saturation regimes in the nuclear scattering. Concerning vector meson production, our starting point is the recent work in [25], where 
different meson wavefunctions and dipole cross sections are considered for the proton case. It is worth mentioning that although light meson photoproduction is a soft process by definition, it is consistently described in the QCD color dipole picture where there is a suitable model for the soft-hard transition, as occurring in the saturation model.

In order to compare the saturation approach with a successful non-perturbative formalism, we consider a Regge inspired model given by the dipole pomeron framework [26]. The reason for this particular choice is that in this model the soft pomeron having an intercept equal to one; thus it does not violate unitarity for hadron-hadron and vector meson production at higher energies. Moreover, it describes with good agreement the hadronic cross section and even DIS data in a wide range of photon virtualities [27]. For meson production on proton targets, our starting point is the recent work of [28], where exclusive photoproduction by real and virtual photons is described with good agreement. The extension to nuclei is provided by the assumption of vector meson dominance and the Glauber-Gribov formalism.

This paper is organized as follows. In the next section, we present a brief review of the exclusive meson production in the color dipole picture for proton target and its extension to the nuclear case. For the dipole-proton (nucleus) cross section we have considered the phenomenological saturation model, which is briefly described. In Sect. 3, the dipole pomeron parameterization for vector meson photoproduction is presented and its extension to nuclear targets is considered. The results coming out from both models are presented and discussed in Sect.4. Finally, in Sect. 5 we summarize our conclusions.

\section{Vector meson production in the color dipole approach}

Let us introduce the main formulas concerning the vector meson production in the color dipole picture. First, we consider the scattering process $\gamma p \rightarrow V p$, where $V$ stands for both light and heavy mesons. Further, one extends this approach to the nuclear case. The scattering process can be seen in the target rest frame as a succession in time of three factorizable subprocesses:

(i) the photon fluctuates in a quark-antiquark pair (the dipole),

(ii) this color dipole interacts with the target and

(iii) the pair converts into vector meson final state. Using as kinematic variables the $\gamma^{*} N$ CMS energy squared $s=$ $W_{\gamma N}^{2}=(p+q)^{2}$, where $p$ and $q$ are the target and the photon momenta, respectively, the photon virtuality squared $Q^{2}=$ $-q^{2}$ and the Bjorken variable $x=Q^{2} /\left(W_{\gamma N}^{2}+Q^{2}\right)$, the corresponding imaginary part of the amplitude at zero momentum transfer reads [29]

$$
\begin{aligned}
& \operatorname{Im} \mathcal{A}(\gamma p \rightarrow V p) \\
& =\sum_{h, \bar{h}} \int \mathrm{d} z \mathrm{~d}^{2} \boldsymbol{r} \Psi_{h, \bar{h}}^{\gamma}\left(z, \boldsymbol{r}, Q^{2}\right) \sigma_{\operatorname{dip}}^{\operatorname{target}}(\tilde{x}, \boldsymbol{r}) \Psi_{h, \bar{h}}^{V *}(z, \boldsymbol{r}),
\end{aligned}
$$

where $\Psi_{h, \bar{h}}^{\gamma}(z, \boldsymbol{r})$ and $\Psi_{h, \bar{h}}^{V}(z, \boldsymbol{r})$ are the light-cone wavefunctions of the photon and vector meson, respectively. The quark and antiquark helicities are labeled by $h$ and $\bar{h}$ and reference to the meson and photon helicities is implicitly understood. The variable $\boldsymbol{r}$ defines the relative transverse separation of the pair (dipole) and $z(1-z)$ is the longitudinal momentum fractions of the quark (antiquark). The basic blocks are the photon wavefunction, $\Psi^{\gamma}$, the meson wavefunction, $\Psi_{\mathrm{T}, \mathrm{L}}^{V}$, and the dipole-target cross section, $\sigma_{\text {dip }}^{\text {target }}$

In the dipole formalism, the light-cone wavefunctions $\Psi_{h, \bar{h}}(z, \boldsymbol{r})$ in the mixed representation $(r, z)$ are obtained through two dimensional Fourier transform of the momentum space light-cone wavefunctions $\Psi_{h, \bar{h}}(z, \boldsymbol{k})$ (see more details, e.g., in $[1,4,25])$. The normalized light-cone wavefunctions for longitudinally (L) and transversely (T) polarized photons are given by [30]

$$
\begin{gathered}
\Psi_{h, \bar{h}}^{\mathrm{L}}(z, \boldsymbol{r})=\sqrt{\frac{N_{c}}{4 \pi}} \delta_{h,-\bar{h}} e e_{f} 2 z(1-z) Q \frac{K_{0}(\varepsilon r)}{2 \pi}, \\
\Psi_{h, \bar{h}}^{\mathrm{T}(\gamma= \pm)}(z, \boldsymbol{r}) \\
= \pm \sqrt{\frac{N_{c}}{2 \pi}} e e_{f}\left[\mathrm{ie}^{ \pm \mathrm{i} \theta_{r}}\left(z \delta_{h \pm, \bar{h} \mp}-(1-z) \delta_{h \mp, \bar{h} \pm}\right) \partial_{r}\right. \\
\left.+m_{f} \delta_{h \pm, \bar{h} \pm}\right] \frac{K_{0}(\varepsilon r)}{2 \pi},
\end{gathered}
$$

where $\varepsilon^{2}=z(1-z) Q^{2}+m_{f}^{2}$. The quark mass $m_{f}$ plays a role of a regulator when the photoproduction regime is reached. Namely, it prevents non-zero argument for the modified Bessel functions $K_{0,1}(\varepsilon r)$ towards $Q^{2} \rightarrow 0$. The electric charge of the quark of flavor $f$ is given by $e e_{f}$.

For vector mesons, the light-cone wavefunctions are not known in a systematic way and they are thus obtained through models. The simplest approach assumes the same vector current as in the photon case, but introducing an additional vertex factor. Moreover, in general the same functional form for the scalar part of the meson light-cone wavefunction is chosen. Here, we follow the analytically simple DGKP approach [30], which is found to describe in good agreement vector meson production as pointed out in [25]. In this particular approach, one assumes that the dependencies on $\boldsymbol{r}$ and $z$ of the wavefunction are factorized, with a Gaussian dependence on $\boldsymbol{r}$. The DGKP longitudinal and transverse meson light-cone wavefunctions are given by $[30]$

$$
\begin{aligned}
& \Psi_{h, \bar{h}}^{V, L}(z, \boldsymbol{r}) \\
& =z(1-z) \delta_{h,-\bar{h}} \frac{\sqrt{\pi} f_{V}}{2 \sqrt{N_{c}} \hat{e}_{f}} f_{\mathrm{L}}(z) \exp \left[\frac{-\omega_{\mathrm{L}}^{2} \boldsymbol{r}^{2}}{2}\right], \\
& \Psi_{h, \bar{h}}^{V, T(\gamma= \pm)}(z, \boldsymbol{r}) \\
& = \pm\left(\frac{\mathrm{i} \omega_{\mathrm{T}}^{2} r \mathrm{e}^{ \pm \mathrm{i} \theta_{r}}}{m_{V}}\left[z \delta_{h \pm, \bar{h} \mp}-(1-z) \delta_{h \mp, \bar{h} \pm}\right]\right. \\
& \left.\quad+\frac{m_{f}}{m_{V}} \delta_{h \pm, \bar{h} \pm}\right)
\end{aligned}
$$


Table 1. Parameters and normalization of the DGKP vector meson light-cone wavefunctions. The results were obtained using quark mass values from the saturation model (see text)

\begin{tabular}{lcccrr}
\hline $\begin{array}{l}V\left(m_{V}\right) \\
\mathrm{MeV}\end{array}$ & $\hat{e}_{V}$ & $\begin{array}{c}f_{V} \\
{[\mathrm{GeV}]}\end{array}$ & $\begin{array}{c}\omega_{\mathrm{T}} \\
{[\mathrm{GeV}]}\end{array}$ & $\mathcal{N}_{\mathrm{T}}$ & $\begin{array}{c}B_{V} \\
{\left[\mathrm{GeV}^{-2}\right]}\end{array}$ \\
\hline$\rho(770)$ & $1 / \sqrt{2}$ & 0.153 & 0.218 & 8.682 & 9.00 \\
$\omega(782)$ & $1 / 3 \sqrt{2}$ & 0.0458 & 0.210 & 10.050 & 10.14 \\
$\phi(1019)$ & $1 / 3$ & 0.079 & 0.262 & 8.000 & 8.92 \\
$J / \Psi(3097)$ & $2 / 3$ & 0.270 & 0.546 & 7.665 & 4.57 \\
\hline
\end{tabular}

$$
\times \frac{\sqrt{\pi} f_{V}}{\sqrt{2 N_{c}} \hat{e}_{f}} f_{\mathrm{T}}(z) \exp \left[\frac{-\omega_{\mathrm{L}}^{2} \boldsymbol{r}^{2}}{2}\right] .
$$

where $\hat{e}_{f}$ is the effective charge arising from the sum over quark flavors in the meson of mass $m_{V}$. The following values stand for $\rho, \omega, \phi$ and $J / \Psi$, respectively: $\hat{e}_{f}=1 / \sqrt{2}$, $1 / 3 \sqrt{2}, 1 / 3$, and $2 / 3$. The coupling of the meson to the electromagnetic current is labeled by $f_{V}^{2}=3 m_{V} \Gamma_{e^{+} e^{-}} / 4 \pi \alpha_{\mathrm{em}}^{2}$ (see Table 1). The function $f_{\mathrm{T}, \mathrm{L}}(z)$ is given by the BauerStech-Wirbel model [31]:

$$
f_{\mathrm{T}, \mathrm{L}}(z)=\mathcal{N}_{\mathrm{T}, \mathrm{L}} \sqrt{z(1-z)} \exp \left[\frac{-m_{V}^{2}(z-1 / 2)^{2}}{2 \omega_{\mathrm{T}, \mathrm{L}}^{2}}\right] .
$$

The meson wavefunctions are constrained by the normalization condition, which contains the hypothesis of a meson composed only of quark-antiquark pairs, and by the electronic decay width $\Gamma_{V \rightarrow e^{+} e^{-}}$. Both conditions are respectively given by $[4,32]$

$$
\begin{aligned}
& \sum_{h, \bar{h}} \int \mathrm{d}^{2} \boldsymbol{r} \mathrm{d} z\left|\Psi_{h, \bar{h}}^{V(\lambda)}(z, \boldsymbol{r})\right|^{2}=1 \\
& \sum_{h, \bar{h}} \int \frac{\mathrm{d}^{2} \boldsymbol{r}}{(2 \pi)^{2}} \frac{\mathrm{d} z}{z(1-z)}\left[z(1-z) Q^{2}+k^{2}+m_{f}^{2}\right] \\
& \quad \times \Psi_{h, \bar{h}}^{V}(k, z) \Psi_{h, \bar{h}}^{\gamma *}(k, z)=e f_{V} m_{V}\left(\varepsilon_{\gamma}^{*} \cdot \varepsilon_{V}\right) .
\end{aligned}
$$

The above constraints when used on the DGKP wavefunction produce the following relations [25]:

$$
\begin{aligned}
& \omega_{\mathrm{L}, \mathrm{T}}=\frac{\pi f_{V}}{\sqrt{2 N_{c}} \hat{e}_{f}} \sqrt{I_{\mathrm{L}, \mathrm{T}}}, \\
& \int_{0}^{1} \mathrm{~d} z z(1-z) f_{\mathrm{L}}(z) \\
& =\int_{0}^{1} \mathrm{~d} z \frac{2\left[z^{2}+(1-z)^{2}\right] \omega_{\mathrm{T}}^{2}+m_{f}^{2}}{2 m_{V}^{2} z(1-z)} f_{\mathrm{T}}(z)=1,
\end{aligned}
$$

where

$$
\begin{aligned}
& I_{\mathrm{L}}=\int_{0}^{1} \mathrm{~d} z z^{2}(1-z)^{2} f_{\mathrm{L}}^{2}(z) \\
& I_{\mathrm{T}}=\int_{0}^{1} \mathrm{~d} z \frac{\left[z^{2}+(1-z)^{2}\right] \omega_{\mathrm{T}}^{2}+m_{f}^{2}}{m_{V}^{2}} f_{\mathrm{T}}^{2}(z) .
\end{aligned}
$$

The relations in (9) come from the normalization condition, whereas the relations in (10) are a consequence of the leptonic decay width constraints. The parameters $\omega_{\mathrm{T}, \mathrm{L}}$ and $\mathcal{N}_{\mathrm{T}, \mathrm{L}}$ are determined by solving (9) and (10) simultaneously. In Table 1 we quote the results for the transverse component, which will be used in our further analysis in the photoproduction case (the longitudinal component does not contribute at $Q^{2}=0$ ). To be consistent with the phenomenological saturation model, which we will discuss further, we have used the quark masses $m_{u, d, s}=0.14 \mathrm{GeV}$ and $m_{c}=1.5 \mathrm{GeV}$. In the case of the $\phi$ meson, we follow [30] and take $m_{s}=m_{u, d}+0.15 \mathrm{GeV}$. We quote [25] for more details in the present approach and its comparison with data for both photo- and electroproduction of light mesons.

Finally, the imaginary part of the forward amplitude can be obtained by putting the expressions for photon and vector meson (DGKP) wavefunctions, (2) and (3) and (4) and (5), into (1). Moreover, summation over the quark/antiquark helicities and the average over the transverse polarization states of the photon should be taken into account. The longitudinal and transverse components are then written as $[25,30]$

$$
\begin{aligned}
\operatorname{Im} \mathcal{A}_{\mathrm{L}}= & \int \mathrm{d}^{2} \boldsymbol{r} \int_{0}^{1} \mathrm{~d} z \sqrt{\alpha_{\mathrm{em}}} f_{V} 2 z^{2}(1-z)^{2} f_{\mathrm{L}}(z) \\
& \times \exp \left[\frac{-\omega_{\mathrm{L}}^{2} \boldsymbol{r}^{2}}{2}\right] Q K_{0}(\varepsilon r) \sigma_{\mathrm{dip}}^{\text {target }}(\tilde{x}, \boldsymbol{r}) \\
\operatorname{Im} \mathcal{A}_{\mathrm{T}}= & \int \mathrm{d}^{2} \boldsymbol{r} \int_{0}^{1} \mathrm{~d} z \sqrt{\alpha_{\mathrm{em}}} f_{V} f_{\mathrm{T}}(z) \exp \left[\frac{-\omega_{\mathrm{T}}^{2} \boldsymbol{r}^{2}}{2}\right] \\
& \times\left\{\frac{\omega_{\mathrm{T}}^{2} \varepsilon r}{m_{V}}\left[z^{2}+(1-z)^{2}\right] K_{1}(\varepsilon r)+\frac{m_{f}^{2}}{m_{V}} K_{0}(\varepsilon r)\right\} \\
& \times \sigma_{\text {dip }}^{\text {target }}(\tilde{x}, \boldsymbol{r}),
\end{aligned}
$$

with $\sigma_{\text {dip }}^{\text {target }}$ being the dipole-proton cross section in the nucleon case and the dipole-nucleus cross section for scattering on nuclei. For the proton case, there is a lot of phenomenology for $\rho$ and $J / \Psi$ production using recent pQCD parameterizations for the dipole-proton cross section [25] or considering non-perturbative QCD calculations based on stochastic QCD vacuum $[33,34]$. In the next subsection, we briefly review the dipole-nucleon (nucleus) case, given by the phenomenological saturation model, which will be considered in our numerical estimates.

In order to obtain the total cross section, we assume an exponential parameterization for the small $|t|$ behavior of the amplitude. After integration over $|t|$, the total cross section for vector meson production by real/virtual photons in the nucleon (proton) case reads

$$
\sigma(\gamma p \rightarrow V p)=\frac{[\operatorname{Im} \mathcal{A}(s, t=0)]^{2}}{16 \pi B_{V}}\left(1+\beta^{2}\right),
$$

where $\beta$ is the ratio of real to imaginary part of the amplitude and $B_{V}$ labels the slope parameter (we refer for the values we have used to Table 1 ). The values considered 
for the slope parameter are taken from the parameterization used in [3]. For the $\rho$ case, we have taken a different value in order to describe simultaneously the $\mathrm{H} 1$ and ZEUS photoproduction data.

In addition, (13) and (14) represent only the leading imaginary part of the positive-signature amplitude, and its real part can be restored using the dispersion relations $\operatorname{Re} \mathcal{A}=\tan (\pi \lambda / 2) \operatorname{Im} \mathcal{A}$. Thus, for the $\beta$ parameter we have used the simple ansatz

$$
\beta=\tan \left(\frac{\pi \lambda_{\text {eff }}}{2}\right)
$$

where

$$
\lambda_{\text {eff }}=\frac{\partial \ln [\operatorname{Im} \mathcal{A}(s, t=0)]}{\partial \ln s},
$$

with $\lambda_{\text {eff }}=\lambda_{\text {eff }}\left(W_{\gamma N}, Q^{2}\right)$ the effective power of the imaginary amplitude, which depends on both energy and photon virtuality. The correction coming from the real part in photoproduction, where only the transverse component contributes, is about $3 \%$ for light mesons and it reaches $13 \%$ for $J / \Psi$ at high energies. It is worth mentioning that a different computation of the $\beta$ parameter, as in [25], produces a larger effect even in the photoproduction case. An additional correction is still required for heavy mesons, like $J / \Psi$. Namely, skewedness effects which take into account the off-forward features of the process (different transverse momenta of the exchanged gluons in the $t$-channel), are increasingly important in this case. Here, we follow the studies in [35], where the ratio of off-forward to forward gluon distributions reads [35]

$$
R_{g}\left(\lambda_{\mathrm{eff}}\right)=\frac{2^{2 \lambda_{\mathrm{eff}}+3}}{\sqrt{\pi}} \frac{\Gamma\left(\lambda_{\mathrm{eff}}+\frac{5}{2}\right)}{\Gamma\left(\lambda_{\mathrm{eff}}+4\right)},
$$

and we will multiply the total cross section by the factor $R_{g}^{2}$ for the heavy meson case.

In the case of nuclear targets, $B_{V}$ is dominated by the nuclear size, with $B \sim R_{A}^{2}\left(R_{A}=1.2 A^{1 / 3} \mathrm{fm}\right.$ is the nuclear radius) and the non-forward differential cross section is dominated by the nuclear form factor, which is the Fourier transform of the nuclear density profile. Here we use the analytical approximation of the Woods-Saxon distribution as a hard sphere, with radius $R_{A}$, convoluted with a Yukawa potential with range $a=0.7 \mathrm{fm}$. Thus, the nuclear form factor reads [9]

$$
\begin{aligned}
& F(q=\sqrt{|t|}) \\
& =\frac{4 \pi \rho_{0}}{A q^{3}}\left[\sin \left(q R_{A}\right)-q R_{a} \cos \left(q R_{A}\right)\right]\left[\frac{1}{1+a^{2} q^{2}}\right],
\end{aligned}
$$

where $\rho_{0}=0.16 \mathrm{fm}^{-3}$.

The photonuclear cross section is given by

$$
\begin{aligned}
& \sigma(\gamma A \rightarrow V A) \\
& =\frac{\left[\operatorname{Im} \mathcal{A}_{\text {nuc }}(s, t=0)\right]^{2}}{16 \pi}\left(1+\beta^{2}\right) \int_{t_{\min }}^{\infty} \mathrm{d} t|F(t)|^{2},
\end{aligned}
$$

with $t_{\min }=\left(m_{V}^{2} / 2 \omega\right)^{2}$, where $\omega$ is the photon energy.
Having introduced the main expressions for computing vector meson production in the color dipole approach, in what follows we present the saturation model and its extension for the scattering on nuclei targets.

\subsection{Dipole-nucleus cross section in the saturation model}

For electron-proton interactions, the dipole cross section $\sigma_{\text {dip }}^{\text {proton }}$, describing the dipole-proton interaction, is substantially affected by the non-perturbative content. There are several phenomenological implementations for this quantity. The main feature of these approaches is that it enables one to match the soft (low $Q^{2}$ ) and hard (large $Q^{2}$ ) regimes in an unified way. In the present work, we follow the quite successful saturation model [22], which interpolates between the small and large dipole configurations, providing color transparency behavior, $\sigma_{\text {dip }} \sim \boldsymbol{r}^{2}$, as $\boldsymbol{r} \rightarrow 0$, and constant behavior, $\sigma_{\text {dip }} \sim \sigma_{0}$, at large dipole separations. The parameters of the model have been obtained from an adjustment to small $x$ HERA data. Its parameter-free application to diffractive DIS has been also quite successful [22] as well as its extension to virtual Compton scattering [36], vector meson production [3, 25] and two-photon collisions [37]. The parameterization for the dipole cross section takes the eikonal-like form [22],

$$
\begin{aligned}
\sigma_{\text {dip }}^{\text {proton }}\left(\tilde{x}, \boldsymbol{r}^{2}\right) & =\sigma_{0}\left[1-\exp \left(-\frac{Q_{\text {sat }}^{2}(\tilde{x}) \boldsymbol{r}^{2}}{4}\right)\right], \\
Q_{\text {sat }}^{2}(\tilde{x}) & =\left(\frac{x_{0}}{\tilde{x}}\right)^{\lambda} \mathrm{GeV}^{2},
\end{aligned}
$$

where the saturation scale $Q_{\text {sat }}^{2}$ defines the onset of the saturation phenomenon, which depends on energy. The parameters, obtained from a fit to the small- $x$ HERA data, are $\sigma_{0}=23.03(29.12) \mathrm{mb}, \lambda=0.288(0.277)$ and $x_{0}=$ $3.04 \cdot 10^{-4}\left(0.41 \cdot 10^{-4}\right)$ for a 3 -flavor (4-flavor) analysis. An additional parameter is the effective light quark mass, $m_{f}=0.14 \mathrm{GeV}$, which plays the role of a regulator for the photoproduction $\left(Q^{2}=0\right)$ cross section, as discussed before. The charm quark mass is considered to be $m_{c}=$ 1.5 GeV. A smooth transition to the photoproduction limit is obtained via the scaling variable [22],

$$
\tilde{x}=\frac{Q^{2}+4 m_{f}^{2}}{Q^{2}+W_{\gamma N}^{2}} .
$$

The saturation model is suitable in the region below $x=$ 0.01 and the large $x$ limit needs still a consistent treatment. Making use of the dimensional-cutting rules, here we supplement the dipole cross section, (20), with a threshold factor $(1-x)^{n_{\text {thres }}}$, taking $n_{\text {thres }}=5$ for a 3 -flavor analysis and $n_{\text {thres }}=7$ for a 4 -flavor one. This procedure ensures a consistent description of heavy quark production at the fixed target data [38].

Let us discuss the extension of the saturation model for the photon-nucleus interactions. Here, we follow the simple procedure proposed in [23], which consists of an extension 
to nuclei of the saturation model discussed above, using the Glauber-Gribov picture [39], without any new parameter. In this approach, the nuclear version is obtained replacing the dipole-nucleon cross section in (1) by the nuclear one,

$$
\begin{aligned}
& \sigma_{\text {dip }}^{\text {nucleus }}\left(\tilde{x}, \boldsymbol{r}^{2} ; A\right) \\
& =2 \int \mathrm{d}^{2} b\left\{1-\exp \left[-\frac{1}{2} T_{A}(b) \sigma_{\text {dip }}^{\text {proton }}\left(\tilde{x}, \boldsymbol{r}^{2}\right)\right]\right\}
\end{aligned}
$$

where $b$ is the impact parameter of the center of the dipole relative to the center of the nucleus and the integrand gives the total dipole-nucleus cross section for a fixed impact parameter. The nuclear profile function is labeled by $T_{A}(b)$, which will be obtained from a 3-parameter Fermi distribution for the nuclear density [40]. The above equation sums up all the multiple elastic rescattering diagrams of the $q \bar{q}$ pair and is justified for a large coherence length, where the transverse separation $r$ of partons in the multiparton Fock state of the photon becomes as good a conserved quantity as the angular momentum, i.e. the size of the pair $r$ becomes an eigenvalue of the scattering matrix. It is important to emphasize that for very small values of $x$ other diagrams beyond the multiple pomeron exchange considered here should contribute (e.g. pomeron loops) and a more general approach for the high density (saturation) regime must be considered. However, we believe that this approach allows us to obtain lower limits of the high density effects at eRHIC and HERA-A. Therefore, at first glance, the region of applicability of this model should be at small values of $x$, i.e. large coherence lengths, and for not too high values of virtualities, where the implementation of the DGLAP evolution should be required. Consequently, the approach is quite suitable for the analysis of exclusive vector meson photoproduction in the kinematical range of the planned lepton-nucleus colliders (eRHIC and HERA-A). Furthermore, it should be noticed that the energy dependence of the cross sections is strongly connected with the semi-hard scale (the saturation momentum scale). Namely, the saturation effects are larger whether the momentum scale is of order or larger than the correspondent size of the vector meson and the energy growth of the cross section is then slowed down.

\section{Vector meson photoproduction in the dipole pomeron framework}

Let us summarize the main features and expressions for the non-perturbative approach given by the dipole pomeron model [26]. This model describes the vector meson exclusive photoproduction [28] data from HERA without need of a pomeron contribution with intercept higher than 1 , thus not violating the Froissart-Martin bound. The picture of the interaction is given by a photon fluctuating into a quark-antiquark pair and further the nucleon (proton) interacts with it through pomeron or secondary reggeon exchange. After that, the pair converts into a vector meson. In general lines, this picture is quite similar to the one for interaction among hadrons in the Regge limit. In particular for photoproduction, the representation of the photon as a hadron is reasonably supported and the Regge pole theory, with a pomeron universal in all hadron-hadron interactions, can be safely used there.

For the pomeron contribution we follow [28] and one considers the dipole pomeron, which gives a very good description of all hadron-hadron total cross sections. As pomerons and secondary reggeons would be universal objects in Regge theory, the corresponding $j$-singularities of photon-proton amplitudes and their trajectories at the photoproduction limit coincide with those appearing in pure hadronic amplitudes. In particular, the restriction on the pomeron intercept implied by the Froissart-Martin bound suggests that it is a more complicated singularity instead of a simple pole having a universal intercept $\alpha_{\mathbb{P}} \geq 1$, which one would apply also to DIS. In the case of a dipole pomeron, it is a double $j$-pole leading to $\sigma_{\text {tot }}^{h h, \gamma h} \propto \ln s$ and unitarity requirements are covered.

Let us consider the usual Mandelstam variables, $s=$ $W_{\gamma N}^{2}=m_{N}^{2}+2 m_{N} \nu-Q^{2}\left(m_{N}\right.$ is the nucleon mass $), t$ (momentum transfer) and defining the scaling variable, $\bar{Q}^{2}=Q^{2}+m_{V}^{2}$. Making use of the latter quantity, the dipole pomeron model can be generalized for virtual external particles. The scattering amplitude is given by the contribution of reggeons at low energies and the dipole pomeron $\left(\alpha_{\mathbb{P}}(t=0)=1\right)$ dominates at higher energies. A simple pole parameterization is used for the $f$-reggeon. The parameters $\alpha_{\mathbb{P}}(t)$ and $\alpha_{\mathbb{R}}(t)$ are universal and do not depend of the reaction, whereas the couplings $g_{i}$, energy scales $s_{0} i$ and slopes $b_{i}$ are functions of the scaling variable and the same for all reactions.

Taking into account the features discussed above, the differential elastic cross section is written as [28]

$$
\begin{aligned}
& \frac{\mathrm{d} \sigma}{\mathrm{d} t}(\gamma p \rightarrow V p) \\
& =4 \pi\left|\mathcal{A}_{\mathbb{P}}\left(s, t ; m_{V}^{2}\right)+\mathcal{A}_{\mathbb{R}}\left(s, t ; m_{V}^{2}\right)\right|^{2},
\end{aligned}
$$

where in the photoproduction case the amplitudes for the secondary reggeons and pomeron contributions are parameterized as [28]

$$
\begin{aligned}
& \mathcal{A}_{\mathbb{P}}\left(W_{\gamma N}^{2}, t ; m_{V}^{2}\right) \\
& =\mathrm{i} g_{0}\left(t ; m_{V}^{2}\right)\left(-\mathrm{i} \frac{W_{\gamma N}^{2}-m_{p}^{2}}{W_{0}^{2}+m_{V}^{2}}\right)^{\alpha_{\mathbb{P}}(t)-1} \\
& \quad+\mathrm{i} g_{1}\left(t ; m_{V}^{2}\right) \\
& \quad \times \ln \left(-\mathrm{i} \frac{W_{\gamma N}^{2}-m_{p}^{2}}{W_{0}^{2}+m_{V}^{2}}\right)\left(-\mathrm{i} \frac{W_{\gamma N}^{2}-m_{p}^{2}}{W_{0}^{2}+m_{V}^{2}}\right)^{\alpha_{\mathbb{P}}(t)-1}, \\
& \mathcal{A}_{\mathbb{R}}\left(W_{\gamma N}^{2}, t ; m_{V}^{2}\right) \\
& =\mathrm{i} g_{\mathbb{R}}\left(t ; m_{V}^{2}\right)\left(-\mathrm{i} \frac{W_{\gamma N}^{2}-m_{p}^{2}}{W_{0}^{2}+m_{V}^{2}}\right)^{\alpha_{\mathbb{R}}(t)-1},
\end{aligned}
$$

where one takes a linear pomeron trajectory $\alpha_{\mathbb{P}}(t)=$ $1+\alpha_{\mathbb{P}}^{\prime}(0) t$, with the usual value for the slope $\alpha_{\mathbb{P}}^{\prime}(0)=$ 
$0.25 \mathrm{GeV}^{-2}$. The reggeon and pomeron couplings are written as

$$
\begin{aligned}
g_{0,1}\left(t ; m_{V}^{2}\right) & =\frac{g_{0,1} m_{V}^{2}}{\left(W_{0}^{2}+m_{V}^{2}\right)^{2}} \exp \left(b_{\mathbb{P}}^{2} t\right), \\
g_{\mathbb{R}}\left(t ; m_{V}^{2}\right) & =\frac{g_{\mathbb{R}} m_{p}^{2}}{\left(W_{0}^{2}+m_{V}^{2}\right)^{2}} \exp \left(b_{\mathbb{R}}^{2} t\right),
\end{aligned}
$$

where the couplings $g_{0,1}$, the energy scale $W_{0}^{2}\left(\mathrm{GeV}^{2}\right)$ and $t$-slope $b_{\mathbb{P}}^{2}\left(\mathrm{GeV}^{-2}\right)$ for the pomeron are adjustable parameters of the model; in addition $m_{p}^{2}$ is the proton mass. The notation $V$ stands for $\rho, \phi$ and $J / \psi$, whereas $\mathbb{R}=f, \pi$ for $\omega$. The remaining constants for the reggeons, $g_{f}, g_{\pi}, b_{\mathbb{R}}^{2}\left(\mathrm{GeV}^{-2}\right)$ are also adjustable parameters. One uses the same slope $b_{\mathbb{R}}^{2}$ for $f$ and $\pi$ reggeon exchanges. We refer to [28] for details on the fit procedure and the tables for the fitted parameters. Let us stress that the only variable that differentiates among the various vector meson elastic cross sections is the mass of the vector mesons.

Following [28], some additional comments are in order. The behavior in the threshold region is given by multiplying the amplitudes by the correction factor $(1-\tilde{x})^{\delta}$, where $\tilde{x}=\left(m_{p}+m_{V}\right)^{2} / W_{\gamma N}^{2}$ and $\left(m_{p}+m_{V}\right)$ is the reaction threshold. The power $\delta=\sqrt{m_{V}^{2} / m_{0}^{2}}$ drives the energy dependence in that region, with $m_{0}^{2}\left(\mathrm{GeV}^{2}\right)$ fitted from the data. Concerning details in [28] when taking into account the secondary reggeons, for the $\rho, \phi$ and $J / \psi$ meson photoproduction the scattering amplitude was written as the sum of a pomeron and $f$ contribution. Although according to the Okubo-Zweig rule, the $f$ meson contribution ought to be suppressed in the production of $\phi$ and $J / \psi$ mesons, the $f$ meson contribution was added even in the $J / \psi$ meson case. For $J / \psi$, it was found to be negligible whereas it is sizable for $\phi$ meson production.

The $\gamma p \rightarrow V p$ process can be used as input in the calculations of the total cross section for the reaction $\gamma A \rightarrow V A$. A major simplification comes from the use of vector meson dominance, which allows one to relate this photoproduction cross section to the cross section for the forward elastic $V p \rightarrow V p$ scattering. Following vector meson dominance $[41,42]$,

$$
\left.\frac{\mathrm{d} \sigma(\gamma p \rightarrow V p)}{\mathrm{d} t}\right|_{t=0}=\left.\frac{4 \pi \alpha_{\mathrm{em}}}{f_{V}^{2}} \frac{\mathrm{d} \sigma(V p \rightarrow V p)}{\mathrm{d} t}\right|_{t=0},
$$

where $t$ is the squared 4-momentum transfer between the proton and vector meson, $\alpha_{\mathrm{em}}$ is the electromagnetic coupling constant and $f_{V}$ is the vector meson-photon coupling, $f_{V}=4 \pi m_{V} \alpha_{\mathrm{em}}^{2} /\left(3 \Gamma_{V \rightarrow e^{+} e^{-}}\right)$, with $m_{V}$ the vector meson mass and $\Gamma_{V \rightarrow e^{+} e^{-}}$the leptonic decay partial width. Values for $f_{V}^{2} / 4 \pi$ are given in Table II of [9]. Using the optical theorem, the total cross section is given by

$$
\sigma_{\text {tot }}(V p \rightarrow V p)=\left[\left.16 \pi \frac{\mathrm{d} \sigma(V p \rightarrow V p)}{\mathrm{d} t}\right|_{t=0}\right]^{\frac{1}{2}} .
$$

The scattering cross section from heavy nuclei can be found by a (quantum mechanical) Glauber-Gribov calculation,

$$
\sigma_{\text {tot }}(V A \rightarrow V A)
$$

$$
=2 \int \mathrm{d}^{2} b\left[1-\exp \left(-\frac{1}{2} T_{A}(b) \sigma_{\text {tot }}(V p \rightarrow V p)\right)\right] .
$$

As referred to before, the nuclear profile function is labeled by $T_{A}(b)$, which will be obtained from a 3-parameter Fermi distribution for the nuclear density [40]. The optical theorem for nucleus $A$ and vector meson dominance are then used to find the following relation:

$$
\left.\frac{\mathrm{d} \sigma(\gamma A \rightarrow V A)}{\mathrm{d} t}\right|_{t=0}=\frac{\alpha_{\mathrm{em}}}{4 f_{V}^{2}} \sigma_{\mathrm{tot}}^{2}(V A \rightarrow V A) .
$$

From this equation one can directly understand the $A$ dependence in two limiting cases: in the transparent limit there is a $A^{2}$ behavior (typical of coherent processes) and in the black disc limit we have an $A^{\frac{4}{3}}$ rise with the nuclear number $A$. The total photonuclear cross section is then given by

$$
\begin{aligned}
& \sigma_{\text {tot }}(\gamma A \rightarrow V A) \\
& =\left.\frac{\mathrm{d} \sigma(\gamma A \rightarrow V A)}{\mathrm{d} t}\right|_{t=0} \int_{t_{\min }}^{\infty} \mathrm{d} t|F(t)|^{2},
\end{aligned}
$$

where $F(t)$ is given in (18). As $F(t)$ is $A$-dependent we have that integration over $t$ yields a factor of $A^{-\frac{2}{3}}$, which implies a $A^{\frac{4}{3}}\left(A^{\frac{2}{3}}\right)$ behavior in the transparent (black disc) limit.

\section{Nuclear vector meson exclusive photoproduction}

In this section we compute the nuclear cross section for the exclusive photoproduction of vector mesons. Here, we compare the QCD approach given by the saturation model extended to nuclei targets within the color dipole picture as well as a non-perturbative approach rendered by the dipole pomeron model, which does also not violate unitarity at high energies. We focus on the energy range and nuclei targets expected for the future lepton-nuclei colliders (eRHIC and eHERA) and also for the available range to be covered in ultraperipheral heavy-ion collisions (UPC's) at LHC.

In Figs. 1 and 2 are shown the results for the $\rho, \omega, \phi$ and $J / \Psi$ photoproduction cross section as a function of energy for different nuclei, including the proton case. The results in the pQCD and Regge approaches present a mild growth on $W_{\gamma N}$ at high energies stemming from the high energy behavior of the models, whereas the low energy region is consistently described through the threshold factor. For the proton, the experimental data from the DESY-HERA collider [43-48] are also included for the sake of comparison. We can see that the $\rho, \omega$ and $\phi$ results vary only slowly with energy, in contrast with the $J / \Psi$ predictions. The saturation model (solid lines) gives a flatter energy dependence in comparison with the dipole pomeron model (dashed lines). On the other hand, for $J / \Psi$ the situation changes, where the dipole pomeron model produces a mild increasing at high energies in comparison with the saturation model. 

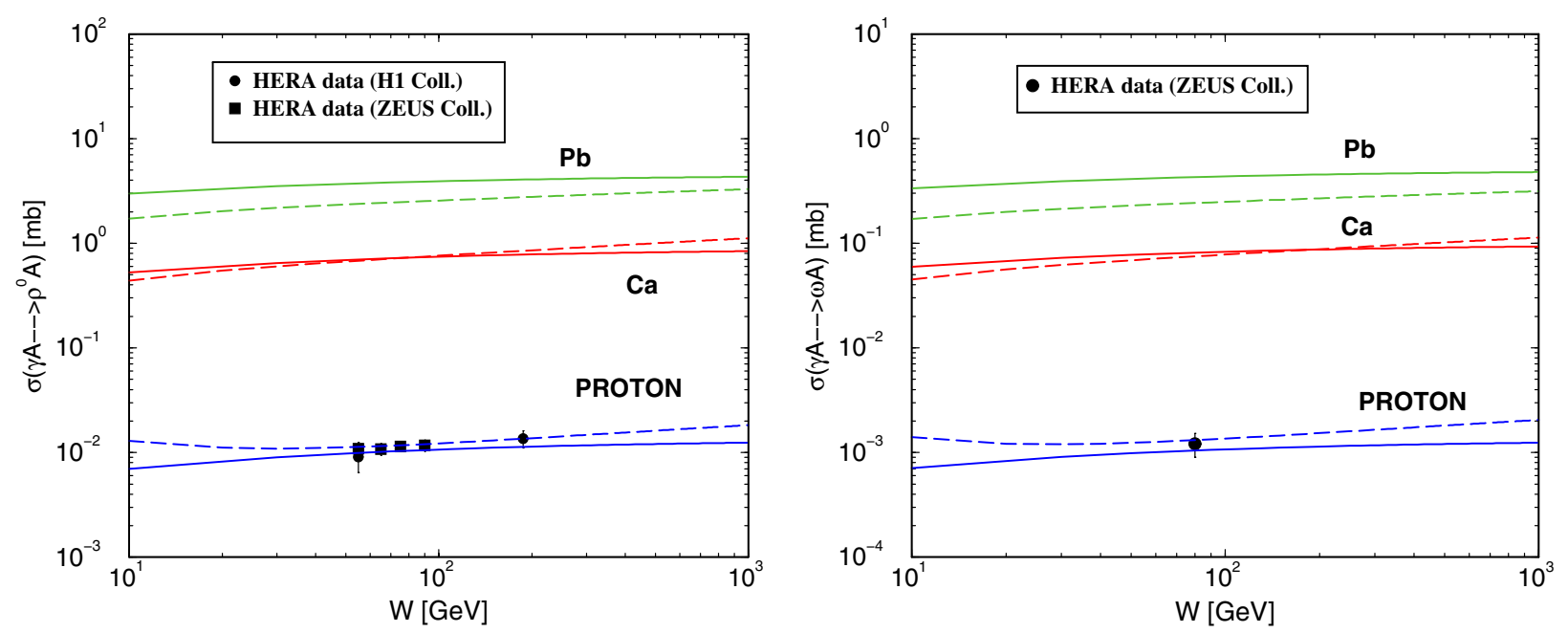

Fig. 1. The total cross section for $\rho$ and $\omega$ photoproduction on proton as well as for light $(\mathrm{Ca})$ and heavy (Pb) nuclei. The solid lines stand for the QCD color dipole approach and the dashed ones for the soft dipole pomeron approach. Experimental high energy data from the DESY-HERA collider on proton targets are also shown
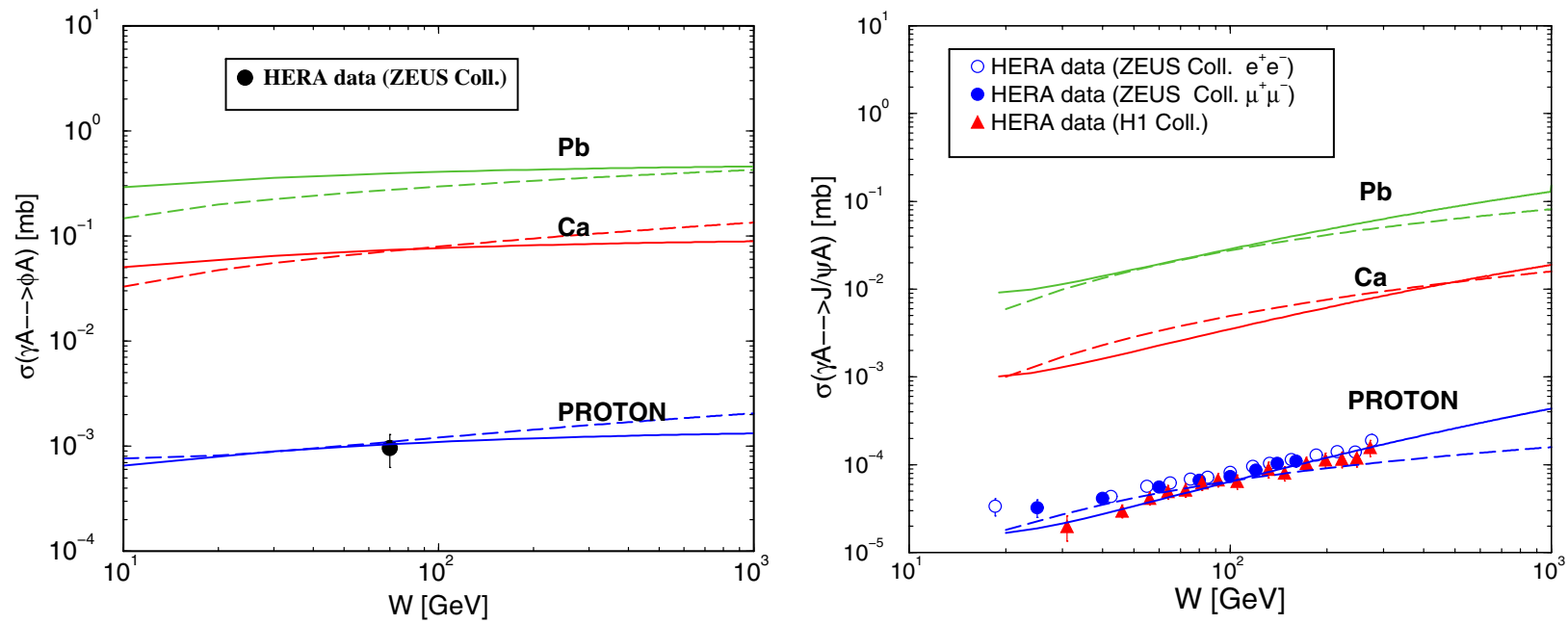

Fig. 2. The total cross section for $\phi$ and $J / \Psi$ photoproduction on protons as well as light (Ca) and heavy (Pb) nuclei. The solid lines stand for the QCD color dipole approach and the dashed ones for the soft dipole pomeron approach. Experimental high energy data from the DESY-HERA collider on proton targets are also shown

Let us discuss the results coming from the color dipole approach. For the proton case we have used (14) and (15) and the dipole-proton cross section given by (20). The contribution of the real part of amplitude is small for the light mesons, whereas it is sizeable for the $J / \Psi$ case. Moreover, the skewedness correction to the $J / \Psi$ photoproduction is important, providing a larger overall normalization, as discussed in Sect. 2. These features remain in the nucleus case, where we have used (19) and the dipole-nucleus cross section given by (22). It is worth mentioning that the effective power of the imaginary part of the amplitude is slowed down in the nuclear case and this has implications in the corrections of the real part and skewedness. The results for photonuclear production on nuclei are consistent with the studies in [9], except for $J / \Psi$, once the growth on energy is mild at high energies in the present case. The agreement with the proton data is consistent and the extension to
$\mathrm{Ca}$ and $\mathrm{Pb}$ targets is suitable since it is constrained by the DESY-HERA data and the validity of the model in the energy range considered. Furthermore, the present investigation is complementary to the ones on heavy quark production [24] and nuclear structure functions [23] using the saturation model for nuclear targets.

For light mesons production in $\gamma p$ processes, the dipole pomeron model predicts a larger growth with the energy than the saturation model due to the dominance of large $q \bar{q}$ pair separations in the saturation approach. This implies that $\sigma_{\text {dip }} \propto \sigma_{0}$, i.e. it is almost energy independent. It can be checked that the integration over dipole sizes in this dipole configuration gives an almost constant value, without logarithmic corrections as in the DIS case. Differently, the dipole pomeron model predicts a logarithmic dependence in the energy. In the nuclear case, this behavior implies a larger modification of the cross section in the 
dipole pomeron model in comparison with the saturation model. In particular, we have that for the nuclear exclusive vector meson photoproduction with $A=\mathrm{Pb}$ we predict that the difference between the results of the models should be a factor of about 1.5. In contrast, for $J / \Psi$ photoproduction, we see that the saturation model predicts a larger cross section for high energies. This is associated to the color transparency regime, present due to the small pair separation between charm and anti-charm. In this case we have a power-like behavior in contrast with the logarithmic dependence present in the dipole pomeron model.

A final comment on the small- $t$ approximation considered here is in order. As shown in [49], the saturation effects play an important role in the $t$-dependence of the scattering amplitude, mostly at large $t$. Therefore, the approximation presented here should be justified. Our master equation is exactly the same as in $[4,49]$, which reads

$$
\begin{aligned}
& \frac{\mathrm{d} \sigma_{\mathrm{L}, \mathrm{T}}}{\mathrm{d} t} \\
& =\frac{1}{16 \pi}\left|\int \mathrm{d}^{2} \mathbf{r} \int \frac{\mathrm{d} z}{4 \pi} \int \mathrm{d}^{2} b\left(\Psi_{V} \Psi_{\gamma}\right)_{\mathrm{L}, \mathrm{T}} \mathrm{e}^{-\mathrm{ib} \cdot \boldsymbol{\Delta}} \frac{\mathrm{d} \sigma_{q q}}{\mathrm{~d}^{2} b}\right|^{2},
\end{aligned}
$$

where the squared momentum transfer is denoted by $\Delta^{2}=$ $-t$. Basically, our expressions (19) and (32) stand for the differential cross section at $t=0$, and further we have used the standard approximations for the small $t$ behavior of the scattering amplitude on nucleon (proton) and nuclei. Namely, for the proton case one has considered the usual exponential parameterization which includes the meson slope parameter $B_{V}$. For the scattering on nuclei, this is accounted for by the nuclear form factor $F_{1}(t)$, which includes the correct size of the nuclear target. Considering the saturation model, the $q \bar{q}$ differential cross section is given by

$$
\frac{\mathrm{d} \sigma_{q \bar{q}}(x, \boldsymbol{r}, b)}{\mathrm{d}^{2} b}=2\left[1-\exp \left(-\frac{1}{2} \sigma_{\text {dip }}(x, \boldsymbol{r}) T(b)\right)\right] .
$$

The remaining issue is what the accuracy is of such an approximation concerning the saturation region for DIS with a nucleon/nucleus target. This can be addressed by looking at the Fourier transform of the differential dipole cross section for large dipole sizes (saturation limit). It was shown in [49] [see Figs. (16) and (17) in that paper] that the saturation effects predict diffractive dips at large $t$. However, the $\mathrm{pQCD}$ picture remains quite the same at small $t$. Therefore, we believe that the integration on $t$ of the complete expression, (33), should not be strongly sensitive to the large $t$ region, once it is sub-dominant in the whole integrand. That is, we expect that the effect on the total cross section is hidden in the integration, whereas it is important at large $t$ in the differential cross section.

\section{Summary and conclusions}

In this paper we have calculated the nuclear cross sections for exclusive vector meson photoproduction within the QCD color dipole picture and Regge approach. These models predict a cross section which does not violate the Froissart-Martin bound at high energies. Since they describe reasonably the experimental data for nucleon (proton) target, we are confident in extending these models to the nuclear photoproduction case.

For the first approach, we have considered the saturation model, which is analytically simple and gives a good description of inclusive and diffractive ep experimental data. This model should be valid until the full non-linear evolution effects become important, which implies the consideration of the pomeron loops beyond the multiple scattering on single nucleons estimated in the present framework. We have verified that the energy behavior is mild, mostly for $J / \Psi$ where we would expect a hard behavior. This means that an important contribution also comes from large dipole configurations, related to the soft domain. We predict absolute values for the cross section being rather large, about $4 \mathrm{mb}$ and $0.12 \mathrm{mb}$ for $\rho$ and $J / \Psi$, respectively, for lead at $W_{\gamma N} \approx 1 \mathrm{TeV}$. These values are similar to those resulting from [9], except for the mild energy behavior for the $J / \Psi$ case presented here. Concerning the $A$-dependence, we have found a behavior proportional to $A^{2 / 3}\left(A^{4 / 3}\right)$ for the cross sections of light (heavy) mesons, in agreement with the theoretical expectations associated with a transition to the black disc regime. In fact, for light mesons the nuclear shadowing in the scattering amplitude is stronger than a simple $A^{1 / 3}$ ansatz, as discussed in [23] when computing the nuclear structure functions. Namely, the saturation scale, which drives the $A$-dependence, between the proton and central nucleus is not simply $\propto A^{1 / 3}$, but has a prefactor which makes the result smaller.

The results presented here can be contrasted, at least for the $J / \Psi$ case, with the results of [50] (hereafter the GLLMN model), where the vector meson production (including DIS production) has been addressed in the color dipole picture. That analysis considers the Glauber approach and a numerical solution of the Balitsky-Kovchegov non-linear evolution equation for the imaginary part of the dipolenucleon scattering amplitude [51]. The comparison is shown in Fig. 3, where the solid lines represent the results from the saturation model (SAT-MOD) and the dashed lines are the numerical result of the GLLMN model. For the proton case (left panel), the saturation model gives a steeper growth on energy, whereas GLLMN produces a mild behavior at large energies. The upper/lower GLLMN curves stand for maximum and minimum values for the total cross section, obtained considering two different values for the correction factor $K_{F}$ (we refer to [50] for more details). It should be noticed that the behavior near threshold is also different in the two models. For the nucleus case (right panel), the behavior on energy remains basically the same as for the proton. The GLLMN model gives a slightly lower cross section at large energies, which is about $8 \%$ smaller than the result for the saturation model at $W_{\gamma A} \approx 400 \mathrm{GeV}$ and presents a flatter behavior on energy. These features are directly associated with the different dipole-nucleon cross sections used in the two approaches. However, considering the relative errors of order of $15-20 \%$ in the predictions from [50], as stated by the authors, we can conclude that 

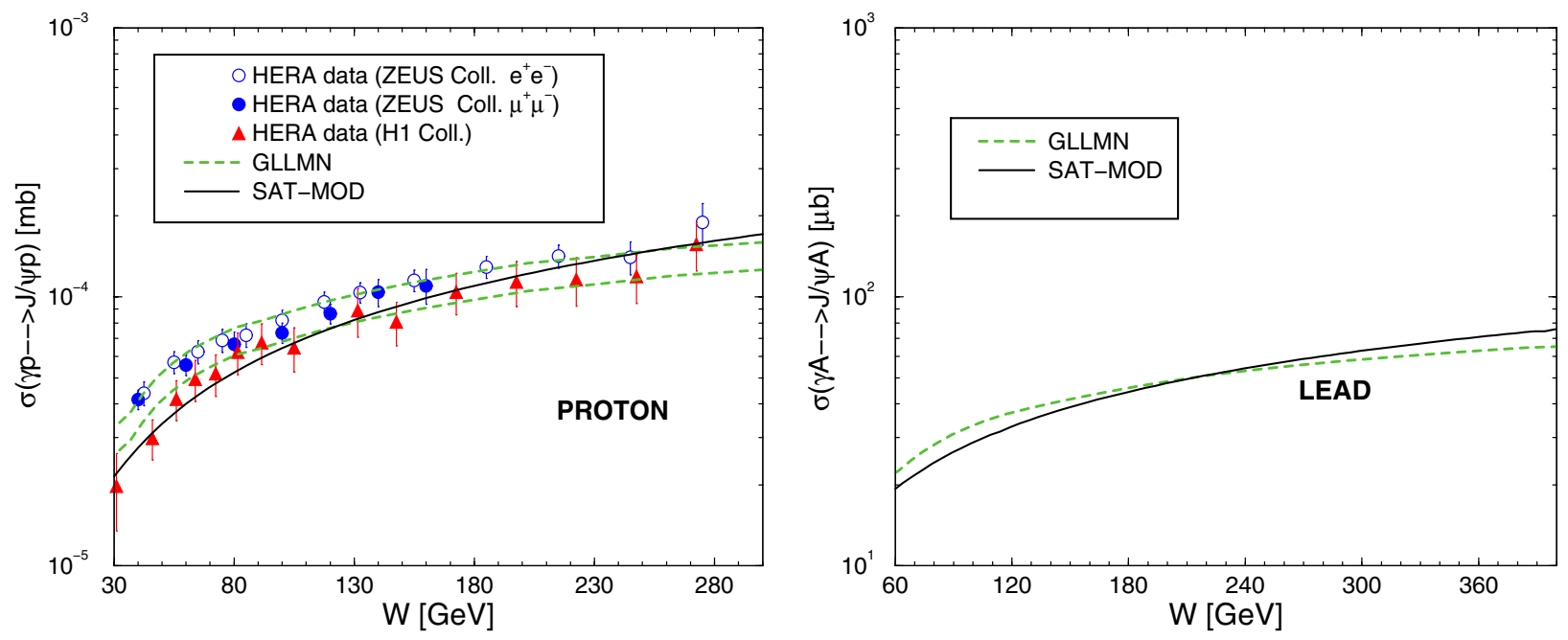

Fig. 3. The total cross section for $J / \Psi$ photoproduction for a proton target (left panel) and for a lead nucleus (right panel). The solid lines stand for the saturation model (SAT-MOD) and the dashed ones for the GLLMN model [50]

the agreement between the results is satisfactory, despite the distinct approximations made in the calculations.

Concerning the non-perturbative approach, we have considered an unitarized pomeron model and computed consistently its extension for nuclear targets. The energy dependence is logarithmic in any case, and the nuclear effects seem to be stronger than in the saturation model for heavy nuclei. The nuclear dependence follows a similar behavior as for the saturation model. However, it should be noticed that we have used a quantum mechanical GlauberGribov calculation for nuclei targets in contrast with the results presented in [9]. As pointed out in [52], the present procedure gives a cross section higher than the classical mechanical model used for the predictions in [9]. For instance, in [52] has been found a difference by a factor 2.5 in $\rho$ photoproduction at RHIC energies.

Our results demonstrate that the experimental analyses of nuclear exclusive vector meson photoproduction in the future electron-nucleus colliders eRHIC and HERA-A could be useful to discriminate between the different theoretical scenarios, mainly if heavy nuclei are considered. An alternative until these colliders become reality is the possibility of using ultraperipheral heavy ion collisions as a photonuclear collider and study vector meson production in this process. Moreover, such processes can also be studied outside the heavy ion mode. For instance, in [53] the photoproduction of heavy vector mesons in $p \bar{p}$ collisions at the Fermilab Tevatron and in the $p p$ collisions at CERN LHC have been discussed, since energetic protons also have large electromagnetic fields. These photoproduction reactions probe the gluon distribution in the proton at very small $x$ values [54], which open a new window to study parton saturation effects in exclusive processes. In a separated publication we will study these possibilities, considering the approaches discussed in this work.

Acknowledgements. The authors are grateful to Ruben Sandapen (Département de Physique et d'Astronomie, Université de Moncton) for his invaluable help and comments. Special thanks go to Michael Lublinsky (DESY Theory Group, DESY) and Eran Naftali (Tel Aviv University, Tel Aviv) for providing us with the numerical results of the GLLMN model for the $J / \Psi$ photoproduction cross section. Useful discussions and valuable help of Igor Ivanov (Sobolev Institute of Mathematics, Novosibirsk State Un.) at early stages of this work are gratefully acknowledged. The authors also thank Prof. Nikolai Nikolaev (IKP, Forschungszentrum Juelich and L.D. Landau Institute) for calling our attention to the pioneering papers on vector meson production within the color dipole approach and their further developments. One of us (M.V.T.M.) thanks for the support of the High Energy Physics Phenomenology Group, GFPAE IF-UFRGS, Brazil. This work was partially financed by the Brazilian funding agencies CNPq and FAPERGS.

\section{References}

1. V. Barone, E. Predazzi, High-energy particle diffraction (Springer-Verlag, Berlin Heidelberg 2002)

2. J.A. Crittenden, Exclusive production of neutral vector mesons at the electron proton collider HERA (SpringerVerlag, Berlin Heidelberg 1997) [hep-ex/9704009]

3. A.C. Caldwell, M.S. Soares, Nucl. Phys. A 696, 125 (2001)

4. S. Munier, A.M. Stasto, A.H. Mueller, Nucl. Phys. B 603, 427 (2001)

5. L.V. Gribov, E.M. Levin, M.G. Ryskin, Phys. Rept. 100, 1 (1983)

6. E. Gotsman, E. Levin, U. Maor, Nucl. Phys. B 464, 251 (1996)

7. M. Arneodo et al., in Proceedings of the workshop on Future Physics at HERA, edited by G. Ingelman, A. De Roeck, R. Klanner, vol. 2, pp. 887-926 (1996), hep-ph/9610423

8. R. Venugopalan, AIP Conf. Proc. 588, 121 (2001) [hepph/0102087]

9. S. Klein, J. Nystrand, Phys. Rev. C 60, 014903 (1999)

10. N.N. Nikolaev, Comments Nucl. Part. Phys. 21, 41 (1992)

11. O. Benhar, B.Z. Kopeliovich, C. Mariotti, N.N. Nicolaev, B.G. Zakharov, Phys. Rev. Lett. 69, 1156 (1992) 
12. B.Z. Kopeliovich, J. Nemchick, N.N. Nikolaev, B.G. Zakharov, Phys. Lett. B 309, 179 (1993)

13. B.Z. Kopeliovich, J. Nemchick, N.N. Nikolaev, B.G. Zakharov, Phys. Lett. B 324, 469 (1994)

14. J. Nemchik, N.N. Nikolaev, B.G. Zakharov, Phys. Lett. B 339, 194 (1994)

15. N.N. Nikolaev, J. Speth, B.G. Zakharov, Phys. Atom. Nucl. 63, 1463 (2000) [Yad. Fiz. 63, 1463 (2000)]

16. O. Benhar, S. Fantoni, N.N. Nikolaev, B.G. Zakharov, J. Exp. Theor. Phys. 84, 421 (1997) [Zh. Eksp. Teor. Fiz. 111, 769 (1997)]

17. I.P. Ivanov, N.N. Nikolaev, W. Schafer, hep-ph/0312170

18. I.P. Ivanov, N.N. Nikolaev, JETP Lett 69, 294 (1999)

19. I.P. Ivanov, N.N. Nikolaev, Acta Phys. Polon. B 33, 3517 (2002)

20. I.P. Ivanov, Phys. Rev. D 68, 032001 (2003)

21. I.P. Ivanov, hep-ph/0304089

22. K. Golec-Biernat, M. Wüsthoff, Phys. Rev. D 59, 014017 (1999); D 60, 114023 (1999)

23. N. Armesto, Eur. Phys. J. C 26, 35 (2002)

24. V.P. Goncalves, M.V.T. Machado, Eur. Phys. J. C 30, 387 (2003)

25. J.R. Forshaw, R. Sandapen, G. Shaw, Phys. Rev. D 69, 094013 (2004)

26. P. Desgrolard, M. Giffon, A. Lengyel, E. Martynov, Nuovo Cim. A 107, 637 (1994)

27. P. Desgrolard, E. Martynov, Eur. Phys. J. C 22, 479 (2001)

28. E. Martynov, E. Predazzi, A. Prokudin, Eur. Phys. J. C 26, 271 (2002); Phys. Rev. D 67, 074023 (2003)

29. N.N. Nikolaev, B.G. Zakharov, Phys. Lett. B 332, 184 (1994); Z. Phys. C 64, 631 (1994)

30. H.G. Dosch, T. Gousset, G. Kulzinger, H.J. Pirner, Phys. Rev. D 55, 2602 (1997)

31. M. Wirbel, B. Stech, M. Bauer, Z. Phys. C 29, 637 (1985)

32. S.J. Brodsky, G.P. Lepage, Phys. Rev. D 22, 2157 (1980)

33. H.G. Dosch, E. Ferreira, Eur. Phys. J. C 29, 45 (2003)

34. H.G. Dosch, E. Ferreira, Phys. Lett. B 576, 83 (2003)
35. A.G. Shuvaev, K.J. Golec-Biernat, A.D. Martin, M.G. Ryskin, Phys. Rev. D 60, 014015 (1999)

36. L. Favart, M.V.T. Machado, Eur. Phys. J. C 29, 365 (2003); C 34, 429 (2004)

37. N. Timneanu, J. Kwiecinski, L. Motyka, Eur. Phys. J. C 23, 513 (2002)

38. C. Brenner Mariotto, M.B. Gay Ducati, M.V.T. Machado, Phys. Rev. D 66, 114013 (2002)

39. V.N. Gribov, Sov. Phys. JETP 29, 483 (1969); 30, 709 (1970)

40. C.W. De Jager, H. De Vries, C. De Vries, Atom. Data Nucl. Data Tabl. 14, 479 (1974)

41. T.H. Bauer, R.D. Spital, D.R. Yennie, F.M. Pipkin, Rev. Mod. Phys. 50, 261 (1978) [Erratum 51, 407 (1979)]

42. F.J. Gilman, Phys. Rept. 4, 95 (1972)

43. J. Breitweg et al. [ZEUS Collaboration], Eur. Phys. J. C 6, 603 (1999)

44. C. Adloff et al. [H1 Collaboration], Eur. Phys. J. C 13, 371 (2000); Nucl. Phys. B 468, 3 (1996)

45. M. Derrick et al. [ZEUS Collaboration], Phys. Lett. B 377, 259 (1996); B 380, 220 (1996)

46. J. Breitweg et al. [ZEUS Collaboration], Phys. Lett. B 487, 273 (2000)

47. C. Adloff et al. [H1 Collaboration], Phys. Lett. B 483, 23 (2000)

48. S. Chekanov et al. [ZEUS Collaboration], Eur. Phys. J. C 24, 345 (2002)

49. H. Kowalski, D. Teaney, Phys. Rev. D 68, 114005 (2003)

50. E. Gotsman, E. Levin, M. Lublinsky, U. Maor, E. Naftali, Acta Phys. Polon. B 34, 3255 (2003); hep-ph/0302010

51. E. Gotsman, E. Levin, M. Lublinsky, U. Maor, Eur. Phys. J. C 27, 411 (2003)

52. L. Frankfurt, M. Strikman, M. Zhalov, Phys. Rev. C 67, 034901 (2003)

53. S.R. Klein, J. Nystrand, Phys. Rev. Lett. 92 , 142003 (2004)

54. V.P. Goncalves, C.A. Bertulani, Phys. Rev. C 65, 054905 (2002) 